\title{
Perfil dos usuários do acolhimento noturno de um CAPS III do Município de São
}

\section{Paulo}

Profile of attendees of the user embracement in a Psychosocial Care Center III in the City of Sao

Paulo

\author{
Perfil de los usuarios del acogimiento nocturno de un Centro de Atención Psicossocial en la Ciudad \\ de Sao Paulo
}

Recebido: 26/07/2021 | Revisado: 31/07/2021 | Aceito: 02/08/2021 | Publicado: 07/08/2021

\author{
Nathalia Nakano Telles \\ ORCID: https://orcid.org/0000-0002-9176-887X \\ Universidade de São Paulo, Brasil \\ E-mail: nnakanot@gmail.com \\ Paula Hayasi Pinho \\ ORCID: https://orcid.org/0000-0001-8922-0699 \\ Universidade Federal do Recôncavo Baiano, Brasil \\ E-mail: paulahpinho@gmail.com \\ Heloísa Garcia Claro \\ ORCID: https://orcid.org/0000-0003-1504-7074 \\ Universidade Estadual de Campinas, Brasil \\ E-mail: clarohg@unicamp.br \\ Márcia Aparecida Ferreira de Oliveira \\ ORCID: https://orcid.org/0000-0002-1069-8700 \\ Universidade de São Paulo, Brasil \\ E-mail: marciaap@usp.br
}

\begin{abstract}
Resumo
Este estudo buscou apresentar o perfil sociodemográfico dos usuários de um CAPS III, da cidade de São Paulo, que necessitaram do acolhimento como estratégia de cuidado no período de novembro de 2012 a dezembro de 2016. Também se analisaram as características dos acolhimentos em relação ao tempo de permanência e acolhimentos repetidos. Trata-se de um estudo descritivo exploratório de análise documental com abordagem quantitativa. Foram analisados o documento preenchido no CAPS referente aos acolhimentos e os prontuários dos usuários. A análise dos dados utilizou o Software Statistical Package for the Social Sciences (SPSS®), Versão 20. Verificou-se que a maioria dos usuários acolhidos era do sexo feminino, de cor branca, com escolaridade até o ensino médio, faixa etária dos 31 aos 59 anos e recebia benefício social. A principal hipótese diagnóstica foi de esquizofrenia, transtornos esquizotípicos e transtornos delirantes. A maioria dos usuários não precisou ficar no acolhimento mais do que cinco vezes e os acolhimentos, na sua maioria, não passaram de sete dias. O estudo contribuiu para compartilhar informações sobre o acolhimento noturno, que é uma importante estratégia de cuidado ainda pouco estudada.
\end{abstract}

Palavras-chave: Acolhimento; Perfil de saúde; Saúde mental; Serviços comunitários de saúde mental.

\begin{abstract}
This study intends to describe the sociodemographic profile of attendees of a Psychosocial Care Center III, in the city of Sao Paulo, who needed the user embracement as a care strategy in the period of time between november of 2012 and december of 2016. This is an explorative descritive study of documental analysis with quantitative approach. The user embracement's document and the medical registers were analysed. The data analysis was done with the Software Statistical Package for the Social Sciences (SPSS), Versão 20. It was verified that the majority of attendees was woman, white skined, studies until high school, age between 31 and 59, receiving social benefit and the main diagnosis was schizophrenia. This study helped to share information about the user embracement, which is an important care strategy still insufficiently studied.
\end{abstract}

Keywords: Embracement; Health profile; Mental health; Community mental health services.

\section{Resumen}

Este estudio pretende describir el perfil sociodemográfico de los usuarios a un Centro de Atención Psicosocial III, en la ciudad de Sao Paulo, que necesitaron el acogimiento del usuario como estrategia de atención en el período de tiempo comprendido entre noviembre de 2012 y diciembre de 2016. Este es un Estudio descriptivo exploratorio de análisis documental con enfoque cuantitativo. Se analizaron el documento de abrazamiento del usuario y los registros médicos. 
El análisis de los datos se realizó con el Software Statistical Package for the Social Sciences (SPSS), Versão 20. Se verificó que la mayoría de los usuarios eran mujeres, de piel blanca, estudios hasta el bachillerato, edad entre 31 y 59 años, beneficiario social y el diagnóstico principal fue la esquizofrenia. Este estudio ayudó a compartir información sobre la aceptación del usuario, que es una estrategia de atención importante que aún no se ha estudiado lo suficiente.

Palabras clave: Acogimiento; Perfil de salud; Salud mental; Servicios comunitarios de salud mental.

\section{Introdução}

Na década de 1970, iniciou-se o movimento da Reforma Psiquiátrica no Brasil decorrente das denúncias do modelo de assistência prestado pelos hospitais psiquiátricos, no contexto da redemocratização do país. O movimento é considerado o processo histórico de formulação crítica e prática que questiona e elabora propostas de transformação do modelo clássico na psiquiatria (Amarante, 2013). No entanto, como é proposto por Bezerra Júnior (2007), é somente com a virada do século que a Reforma Psiquiátrica no Brasil deixou a posição de "proposta alternativa" e consolidou-se como marco fundamental da política de assistência à saúde mental oficial. Pode-se dizer que este marco está relacionado à Lei no . 10.216/01 (Brasil, 2001), deixando o antigo modelo hospitalocêntrico e propondo o cuidado através de recursos extra-hospitalares, territoriais e comunitários.

Em 2002, a Portaria n ${ }^{\circ}$. 336, definiu as características das modalidades de Centro de Atenção Psicossocial (CAPS) em CAPS I, II, III, ad II e i II (Brasil 2002). O grande diferencial do CAPS III é possuir estrutura capaz de oferecer acolhimento noturno e durante os finais de semana aos usuários do serviço. Segundo a Portaria no 854/12 (Brasil, 2012), o procedimento "acolhimento noturno de um paciente em CAPS" é descrito como:

ação de hospitalidade noturna realizada nos CAPS como recurso do projeto terapêutico singular de usuários já em acompanhamento no serviço, que recorre ao seu afastamento de situações conflituosas e vise ao manejo de situações de crise motivadas por sofrimento decorrente de transtornos mentais - incluídos aqueles por uso de álcool e outras drogas e que envolvem conflitos relacionais (...) não deve exceder o máximo de 14 dias (Brasil, 2012).

Conforme definem Dell'Acqua e Mezzina (1991), as situações de crise são reconhecidas como aquelas que apresentam, pelo menos, três dos seguintes parâmetros: grave sintomatologia psiquiátrica aguda; grave ruptura de relações familiares e/ou sociais; recusa de intervenções, mas aceitação do contato com a equipe; recusa de qualquer foma de contato; e situações emergenciais no contexto familiar e/ou social ou, ainda, impossibilidades pessoais de enfrentá-las.

Diante destas situações, o acolhimento noturno e a permanência aos finais de semana são utilizados como mais um dos recursos terapêuticos possíveis, que visam proporcionar a atenção integral aos usuários, podendo ser utilizados para que se evitem ou tornem-se mais graves as crises (Farnettane, 2016). Kinoshita (2009) ressalta que o acolhimento noturno também pode ser pensado como ação precoce e preventiva, ao invés de ser utilizado como última possibilidade possível apenas.

A Organização Mundial da Saúde (OMS) propõe que os dados epidemiológicos em saúde mental devem ser monitorizados e analisados para que os serviços proporcionem o melhor tratamento possível (Ministério da Saúde, 2002). Gallio e Mezzina (1988) salientam que estudos que buscam entender o perfil dos usuários dos serviços de saúde mental fornecem subsídios para que os governantes aloquem verbas para o enfrentamento dos obstáculos que se apresentam à consolidação e articulação da Rede de Atenção Psicossocial.

Deste modo, este estudo buscou descrever o perfil sociodemográfico da população que utilizou o acolhimento noturno como estratégia de cuidado em seu Projeto Terapêutico Singular (PTS) num CAPS III.

\section{Metodologia}

Trata-se de um estudo descritivo e exploratório de abordagem quantitativa. A escolha por esta abordagem se dá por se tratar de um estudo que descreve os dados encontrados no documento do serviço de saúde estudado, assim como relaciona as variáveis encontradas entre si, formulando hipóteses sobre o porquê do perfil dos usuários ser o apresentado, buscando compreender e classificar o processo experimentado por este grupo social (Prodanov, 2013). O presente estudo é exploratório 
por trazer ao pesquisador a possibilidade de aumentar a experiência e conhecimento sobre o assunto (Marconi \& Lakatos, 2010) Utilizou-se como fonte de dados um documento alimentado pela equipe de enfermagem de um CAPS III do município de São Paulo referente ao acolhimento noturno, e assim, buscou-se descrever o perfil dos usuários que utilizaram-no como forma e estratégia de cuidado.

O documento é uma planilha de dados organizada no programa Microsoft Excel®, atualizada diariamente pelo enfermeiro do plantão noturno desde novembro de 2012. Esta tabela produz um relatório mensal sobre como foi a ocupação das camas no acolhimento noturno. Neste documento, constam: nome, idade, sexo, hipótese diagnóstica primária de acordo com a Classificação Internacional de Doenças (CID-10), datas inicial e final do acolhimento, dias de permanência e motivo de alta. Ainda há dados estatísticos referente às taxas de ocupação no serviço: número de camas disponíveis no mês, número de camas ocupadas por mês, taxa de ocupação e média de camas ocupadas no mês.

Foram coletados os dados de novembro de 2012 a dezembro de 2016 do documento supracitado, bem como dados de caracterização dos indivíduos como raça/cor, renda/benefício e escolaridade, coletados dos prontuários dos usuários.

Os dados incompletos ou ausentes nos prontuários foram codificados como "Não informado/ausente".

Atualmente, o CAPS conta com seis leitos para o acolhimento noturno, sendo três leitos femininos e três masculinos. No entanto, durante dez meses - de março de 2016 a dezembro de 2016 - apenas cinco leitos estavam disponíveis, devido a um incêndio acidental que acarretou em uma cama a menos neste período.

Os dados foram coletados em formulário on-line analisados no Software Statistical Package for the Social Sciences (SPSS $\left.{ }^{8}\right)$, Versão 20. Foi realizada uma análise descritiva dos dados, incluindo frequências, médias, medianas, mínimos e máximos, bem como tabelas de contingência. A análise estatística foi feita a partir da construção iterativa dos dados apresentados, sendo analisados e interpretados a fim de elaborar uma explicação para a situação estudada e examinando as relações entre as variáveis apresentadas no estudo (Gil, 2002).

Este estudo cumpriu as exigências éticas de pesquisa com seres humanos, conforme a Resolução 466/12 do Conselho Nacional de Saúde, do Ministério da Saúde. O projeto que deu origem ao estudo foi aprovado pelo Comitê de Ética em Pesquisa da Escola de Enfermagem da Universidade de São Paulo sob parecer CAAE 68489817.8.0000.5392.

\section{Resultados}

No período analisado, ocorreram 983 acolhimentos noturnos utilizados por 178 usuários do CAPS. A tabela 1, apresenta os dados sociodemográficos dos usuários em uma tabela de contingência, calculando as observações em relação à variável sexo. Observa-se que a maioria dos usuários do acolhimento foi do sexo feminino (54,5\%), de cor branca (46,6\%) e com estudos até o ensino médio (28,7\%). A faixa etária dos 31 aos 59 anos abrangeu a maior parte dos usuários em acolhimento (60,7\%). Mais de um quarto dos usuários $(28,7 \%$ ) recebe Benefício de Prestação Continuada (BPC) ou aposentadoria, seguido pelos usuários que não recebem benefício algum e também não tem nenhuma outra fonte de renda própria (21,9\%). Em relação à hipótese diagnóstica atribuída a estes usuários, 45,5\% deles foram diagnosticados com esquizofrenia, transtornos esquizotípicos e transtornos delirantes, seguidos pelo diagnóstico de transtornos de humor $(28,7 \%)$.

Na Tabela 1, é possível observar que, em todos os cruzamentos feitos, as informações ausentes estão sempre em maior número quando as usuárias são do sexo feminino. Há pouca disparidade entre os sexos nos cruzamentos feitos, salvo algumas exceções: o número absoluto de usuários mais jovens, de 20 a 30 anos, acolhidos foi mais que o dobro quando compara-se o sexo masculino com o feminino (15 homens e 7 mulheres); já em relação aos usuários com 60 anos ou mais, estes são na maioria do sexo feminino (17 mulheres e 9 homens). Em relação à hipótese diagnóstica, há maioria absoluta (56,8\%) de usuários homens diagnosticados com esquizofrenia, transtornos esquizotípicos e transtornos delirantes. Nas mulheres, a maioria fica entre este mesmo diagnóstico (36,1\%), seguido dos transtornos de humor (32\%), sendo este número menor no sexo masculino (24,7\%). 
Tabela 1. Cruzamento da variável sexo com as variáveis cor, escolaridade, faixa etária, renda e hipótese diagnóstica e valor total das variáveis.

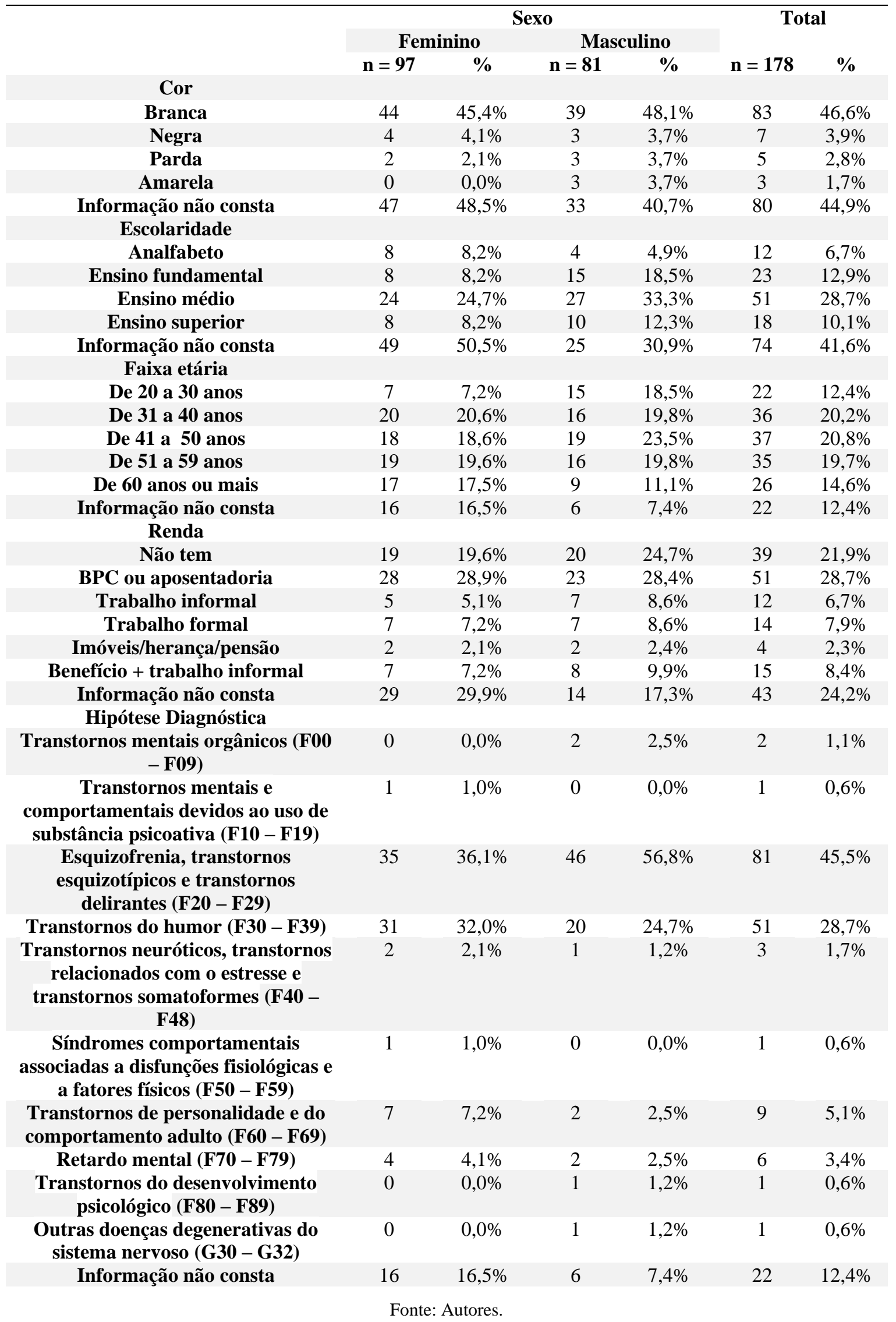


Ao analisar-se o número de acolhimentos repetidos pelo mesmo usuário, temos que este número tende a diminuir conforme maior for a repetição, em ambos os sexos, até a categoria "de 16 a 20 acolhimentos", como consta na Tabela 2 . A maioria dos usuários, tanto homens quanto mulheres, utilizou o acolhimento noturno cinco vezes ou menos (120 usuários, no total).

Tabela 2. Cruzamento entre o número de vezes que o usuário precisou do acolhimento noturno e a variável sexo.

\begin{tabular}{|c|c|c|c|c|c|c|}
\hline & $\begin{array}{c}\text { De } 1 \text { a } 5 \\
\text { acolhimentos }\end{array}$ & $\begin{array}{c}\text { De } 6 \text { a } 10 \\
\text { acolhimentos }\end{array}$ & $\begin{array}{c}\text { De } 11 \text { a } 15 \\
\text { acolhimentos }\end{array}$ & $\begin{array}{c}\text { De } 16 \text { a } 20 \\
\text { acolhimentos }\end{array}$ & $\begin{array}{c}21 \text { ou mais } \\
\text { acolhimentos }\end{array}$ & Total \\
\hline Feminino & 68 & 13 & 8 & 2 & 6 & 97 \\
\hline Masculino & 52 & 18 & 5 & 2 & 4 & 81 \\
\hline Total geral & 120 & 31 & 13 & 4 & 10 & 178 \\
\hline
\end{tabular}

Na Tabela 3, analisa-se cada um dos 983 acolhimentos realizados no período estudado conforme o tempo de permanência no CAPS. Quase 75\% de todos os acolhimentos duraram até sete dias. As mulheres são maioria no acolhimento em todos os períodos até o máximo de 60 dias; quando o período ultrapassa 60 dias, os homens são maioria no acolhimento (62,5\%). Quanto à faixa etária, percebe-se que os mais jovens - de 20 a 30 anos - ficaram menos tempo em acolhimento quando comparados aos adultos mais velhos - a partir dos 31 anos. Os usuários com diagnóstico de esquizofrenia, transtornos esquizotípicos e transtornos delirantes são maioria em todos os períodos de permanência no acolhimento, seguido pelos usuários com diagnóstico de transtornos do humor. 
Tabela 3. Cruzamento do tempo de permanência no acolhimento noturno com as variáveis sexo, faixa etária e hipótese diagnóstica

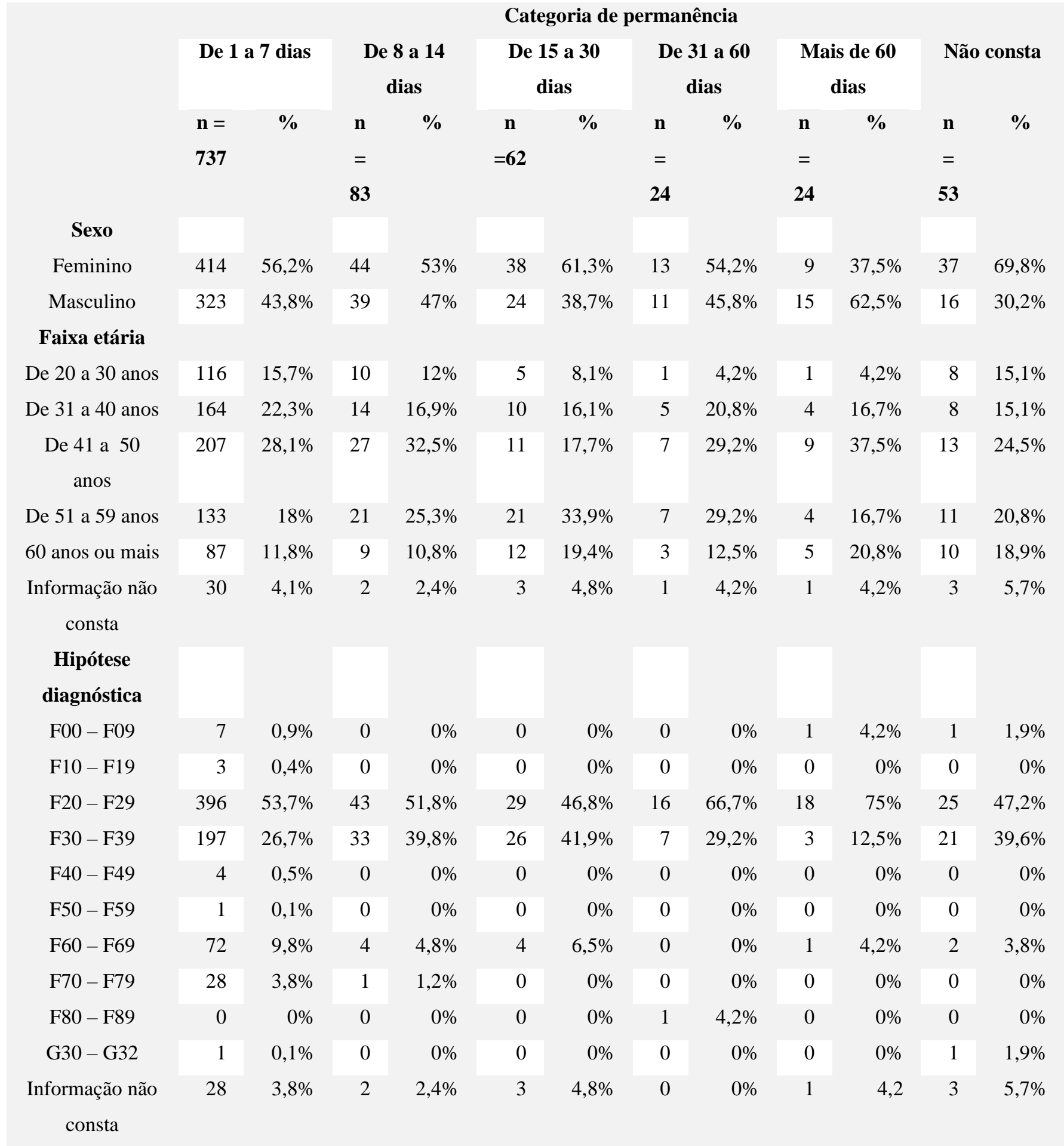

Fonte: Autores.

A Tabela 4 apresenta que a média de dias em acolhimento pelas mulheres (sete dias) é menor que a média dos homens (dez dias) e ainda, que o máximo de dias de acolhimento necessário para um usuário do sexo masculino (291 dias) foi mais que o dobro de dias necessário para a usuária do sexo feminino que precisou do acolhimento por mais tempo (139 dias). Em relação à faixa etária, percebe-se que conforme maior a idade do usuário, maior é o tempo médio que ele necessita no acolhimento. O máximo de dias de acolhimento não apresentou relação direta com a idade. Quanto à fonte de renda, não há relação direta entre 
renda e média de dias de permanência no acolhimento noturno; porém, os usuários que têm como renda o BPC e aposentadoria, foram os que tiveram maior tempo de permanência no acolhimento noturno.

Tabela 4. Dados sobre os dias de permanência conforme as variáveis sexo, faixa etária e renda.

\begin{tabular}{|c|c|c|c|c|c|}
\hline & \multicolumn{5}{|c|}{ Dias de permanência } \\
\hline & Média & Mediana & Minímo & Máximo & Soma \\
\hline \multicolumn{6}{|l|}{ Sexo } \\
\hline Feminino & 7 & 2 & 1 & 139 & 3802 \\
\hline Masculino & 10 & 2 & 1 & 291 & 4140 \\
\hline \multicolumn{6}{|l|}{ Faixa etária } \\
\hline De 20 a 30 anos & 4 & 2 & 1 & 75 & 567 \\
\hline De 31 a 40 anos & 7 & 3 & 1 & 175 & 1428 \\
\hline De 41 a 50 anos & 8 & 2 & 1 & 157 & 2126 \\
\hline De 51 a 59 anos & 10 & 2 & 1 & 139 & 1782 \\
\hline 60 anos ou mais & 14 & 3 & 1 & 291 & 1593 \\
\hline Informação não consta & 12 & 1 & 1 & 261 & 446 \\
\hline \multicolumn{6}{|l|}{ Renda } \\
\hline Não tem & 7 & 2 & 1 & 107 & 1108 \\
\hline BPC ou aposentadoria & 9 & 3 & 1 & 291 & 3876 \\
\hline Trabalho informal & 10 & 2 & 1 & 120 & 425 \\
\hline Trabalho formal & 4 & 3 & 1 & 18 & 146 \\
\hline Imóveis/herança/pensão & 28 & 21 & 1 & 132 & 792 \\
\hline Benefício + Trabalho informal & 6 & 2 & 1 & 157 & 775 \\
\hline Informação não consta & 8 & 2 & 1 & 261 & 820 \\
\hline
\end{tabular}

Fonte: Autores.

A Tabela 5 mostra que mais de $86 \%$ dos acolhimentos teve como motivo de saída a alta do usuário, seguido pela evasão (6,5\% dos casos), transferência para outros serviços de saúde $(0,6 \%)$ e outros motivos $(0,5 \%)$. Os usuários do sexo masculino são maioria apenas quando o motivo de saída foi a transferência para outro equipamento de saúde (66,7\%). A alta foi o motivo de saída predominante em todos os períodos de permanência no acolhimento. A evasão do serviço ocorreu em todos os períodos de acolhimento, mas a maior parte ocorreu quando havia até sete dias de acolhimento (59,4\%). O número de evasões vai diminuindo conforme o período no acolhimento aumenta. 
Tabela 5. Cruzamento da variável motivo de saída do acolhimento com as variáveis sexo e dias de permanência.

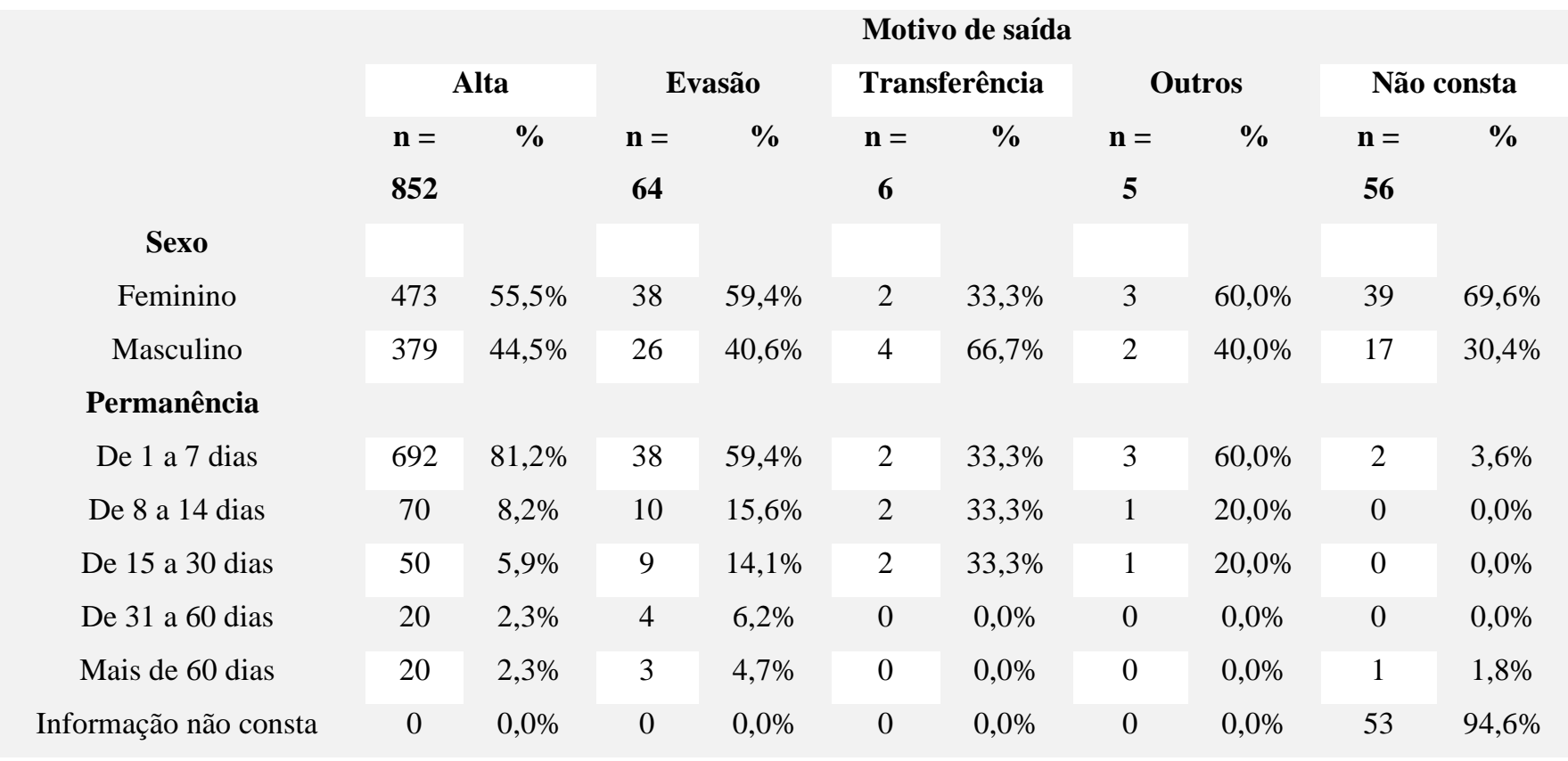

Fonte: Autores.

\section{Discussão}

No período estudado, constatou-se predominância de usuárias do sexo feminino no acolhimento noturno. Não foram encontrados outros estudos sobre caracterização da população que utilizou essa estratégia de cuidado, mas existem estudos que delinearam o perfil sociodemográfico de usuários de CAPS pelo Brasil. Quanto ao sexo dos usuários do CAPS, não houve unanimidade: alguns apontaram haver mais mulheres no serviço (Gallio \& Mezzina, 1988; Brasil, 2002; Santos, Nascimento, Veríssimo, Cavalcante, Brêda \& Holanda, 2013), assim como o presente estudo e outros apontam mais homens nos CAPS (Pelisoli \& Moreira, 2005; Balarin, Miranda \& Carvalho, 2011; Silva, Lima \& Ruas, 2020; Souza \& Padula, 2020). Os dois primeiros estudos citados anteriormente estudaram CAPS que atendem usuários de álcool e outras drogas, o que pode ter levado a este dado, uma vez que a população atendida costuma ser prevalentemente masculina, já que as mulheres têm mais dificuldade de iniciar o tratamento nestes casos devido ao preconceito com mulheres dependentes de álcool e/ou outras drogas, conforme apresentado no estudo de Pelisoli e Moreira (2007).

O estudo de Balarin et al. (2011) apresenta que a maior parte dos CAPS estudados apresentou maioria dos usuários de cor branca; neste estudo, os usuários que foram identificados quanto raça/cor também apresentaram este perfil. Silva, Barros, Azevedo, Batista e Policarpo (2017) trazem um estudo de revisão sistemática sobre como o quesito raça/cor é utilizado em estudos de caracterização de usuários de CAPS, no qual apresentam que dentre os 17 artigos selecionados no estudo delas, apenas três utilizaram tal quesito e nenhum deles discutiu esta variável posteriormente. As autoras ainda apontam a dificuldade que pesquisadores no campo da saúde têm de reconhecer a variável raça/cor como importante para compreensão da determinação social do processo saúde/doença mental e de que a dimensão étnico-racial não é reconhecida como configuração e reprodução das iniquidades sociais. Das variáveis selecionadas neste presente estudo, a variável raça/cor foi a que menos teve registros nos prontuários dos usuários (83 usuários brancos e $80 \mathrm{sem}$ raça/cor informada). Não se sabe como este dado foi coletado nos prontuários - autodeclaração do usuário ou opinião do próprio profissional - mas levanta-se a hipótese de que talvez o número de usuários por raça/cor mude caso todos os prontuários estejam completos neste quesito. É importante ressaltar o comprometimento existente na atuação do serviço quando é deixado de ofertar atendimento qualificado ao usuário desconsiderando sua origem étnico-racial, cor da pele e/ou cultura, sendo essa prática resultante do racismo institucional (Brasil, 
2007). Este é um dado importante, uma vez que qualificar esta informação contribui para a elaboração e promoção de políticas públicas que visam diminuir as desigualdades raciais em saúde.

Em relação à escolaridade, diferentemente dos estudos de Santos et al. (2013), Belletini e Gomes (2013), Costa, Coelho, Neto, Marques e Lotif (2015), Silva et al. (2017), Silva, Lima e Ruas (2020) e Souza e Padula (2020), que indicam que a maioria dos usuários dos CAPS estudou até o ensino fundamental, a maioria dos usuários deste CAPS, que estiveram em acolhimento noturno e em cujos prontuários essa informação constava, estudou até o ensino médio. Esta diferença talvez se dê pelo fato deste CAPS ter como área de abrangência uma das regiões mais ricas da cidade de São Paulo, o que poderia explicar também o número expressivo de usuários que cursou o ensino superior - sendo este número maior do que nos estudos usados como comparativo. Assim como a variável raça/cor, a variável referente ao grau de escolaridade também está ausente em grande número dos prontuários dos usuários. Este dado é de grande importância para traçar a trajetória de vida do usuário e pensar em seu Projeto Terapêutico Singular (PTS), assim como também serve de indicador de estratégia de intervenção individualizada. Este dado, muitas vezes esquecido, é apontado como "variável sombra" por Saraceno (1994), pois apesar de ser esquecido muitas vezes, tem papel fundamental no cuidado dos usuários. Estas variáveis normalmente são deixadas à sombra por serem consideradas irrelevantes na evolução da doença e na estratégia de intervenção junto ao sujeito. No entanto, dois usuários com o mesmo diagnóstico provavelmente terão diferentes prognósticos devido à existência e/ou influência destas variáveis "sombra". O fato de apenas usuários das cores branca e amarela terem cursado ensino superior reflete a desigualdade de acesso ao ensino superior que se encontra no Brasil (Instituto Brasileiro de Geografia e Estatística [IBGE], 2016).

Os usuários de 31 a 59 anos foram os que mais precisaram do acolhimento noturno. Este dado é semelhante a diversos estudos de caracterização do perfil de usuários de CAPS (Santos et al.,2013; Belletini \& Gomes, 2013; Costa et al., 2015; Pereira, Souza, Costa, Vargas, Oliveira \& Moura, 2012; Silva et al., 2017) nos quais os usuários sempre se encontram em idade adulta e produtiva em sua maioria. Os estudos de Costa et al. (2015) e Pereira et al. (2012) ainda trazem que as mulheres que frequentam os CAPS estudados são, na média, mais velhas que os homens, assim como é apresentado neste estudo. Os usuários mais jovens tendem a ficar menos tempo acolhidos do que os mais velhos. Levanta-se aqui a hipótese de este fato ocorrer devido ao maior suporte familiar/social que as pessoas costumam ter quando mais jovens e que vão se desgastando durante o decorrer da vida. As mulheres idosas apresentam-se em maior número no acolhimento do que os homens idosos. Provavelmente, segue-se a lógica do envelhecimento da população, no qual as mulheres têm expectativa de vida superior aos homens (IBGE, 2016). Ttambém traz-se a hipótese de que, considerando que as mulheres são socialmente responsáveis pelo cuidado, ao se tornarem idosas e não terem rede de apoio, as mulheres precisam da ajuda do Estado; enquanto os homens têm rede de apoio para serem cuidados por viverem menos tempo na média.

Em relação à situação de renda dos usuários do acolhimento noturno do CAPS, apenas 23,1\% dos usuários realizavam algum tipo de trabalho remunerado. Este número é inferior ao encontrado no estudo de Silva et al. (2017) e apresenta diferença gritante em relação ao índice de desocupação da população brasileira geral que é de 12,3\% (IBGE, 2018). Notou-se que os únicos usuários que não estavam no mercado de trabalho foram os analfabetos. O baixo grau de escolaridade, a qualificação profissional precária ou a não qualificação profissional dos usuários do CAPS leva-os a trabalhos com mão de obra de fácil substituição e a condições desfavoráveis na competição no mercado de trabalho, o que levará a uma alta taxa de desemprego, visto o mercado altamente competitivo do cenário atual (Silva et al., 2017). Ainda se salienta que ser uma pessoa com transtorno mental grave traz consigo o estigma da doença mental que coloca os usuários e as usuárias de CAPS em desvantagem nas relações sociais de modo geral e em qualquer disputa por emprego em particular, considerando que o poder contratual destas pessoas (Kinoshita, 2016) é posto em xeque em diversas situações e por isso, é tema tão relevante e investido na Reabilitação Psicossocial. Atentase, então, à importância da existência e investimento em empreendimentos solidários e de geração de renda dentro dos CAPS, já que estes apresentam-se como alternativa ao mercado de trabalho competitivo e que consolidam diretrizes da Reforma 
Psiquiátrica, dentre elas "responder à necessidade básica de qualquer cidadão, que é o direito aos bens de consumo, culturais, sociais, imateriais, por meio do trabalho", como afirma Silva (2012, p. 48). Ainda salienta-se que a maioria dos usuários que trabalham é do sexo masculino, o que pode estar relacionada à questão da relação entre o sexo masculino ser o provedor e ter o trabalho como algo fundamental na vida do homem (Zanello, Fiuza \& Costa, 2015) e também sobre a desigualdade de oportunidades de emprego e entre salários quando comparados homens e mulheres, nas quais as pessoas do sexo feminino sempre aparecem em desvantagem (IBGE, 2018).

O CAPS deve ser responsável pelo cuidado das pessoas com transtornos mentais severos e persistentes. Ao levar esta informação em consideração, atenta-se que o CAPS estudado atende, em sua maioria, pessoas com o diagnóstico de esquizofrenia, transtornos esquizotípicos e transtornos delirantes, que causam muitas limitações na vida das pessoas acometidas por esta doença. Este dado é condizente com os obtidos em outros estudos (Santos et al., 2013; Silva et al. 2017), embora outros estudos apontem para a maioria de seus usuários terem o diagnóstico de transtornos de humor mais prevalentes do que os transtornos psicóticos (Belletini \& Gomes, 2013; Costa et al., 2015). No presente estudo, também tem-se o resultado de que os homens são mais diagnosticados na categoria F20 - F29 do que as mulheres, além de estas terem significativo número de diagnósticos na categoria F30 - F39. Os homens aparecem mais propensos à esquizofrenia (Pereira et al., 2012), apesar não haver diferença significativa entre os gêneros quanto ao desenvolvimento de esquizofrenia, enquanto as mulheres parecem ser mais propensas aos transtornos de humor (Santos \& Siqueira, 2010).

Dentre os 50 meses estudados, a maioria dos usuários não precisou mais do que cinco acolhimentos e quase $75 \%$ dos acolhimentos duraram até sete dias, conforme a Portaria GM nº 336/12. No entanto, há um número expressivo de acolhimentos longos e também de "reacolhimentos" repetidas vezes que deve ser analisado de perto. O número de usuários que precisaram do acolhimento diminuiu conforme aumentou o número de repetições no acolhimento. Acredita-se que tal fato ocorreu devido ao investimento feito por este CAPS a cada novo acolhimento atrelando-se outras estratégias de cuidado para que os usuários saíssem da crise e pudessem retornar à sua vida. Comparando os resultados deste estudo com o estudo de Machado e Santos (2011) sobre internações em um hospital psiquiátrico, que trazem dados sobre reinternações em hospital psiquiátrico, é muito importante salientar a diferença da média de dias que usuários tiveram ao ficar nestes serviços. Enquanto no CAPS, a média de permanência das mulheres foi de sete dias e dos homens, dez dias, no hospital psiquiátrico, estes números sobem para 36 e 17,3 dias, respectivamente. Destaca-se aqui a diferença de dias que o CAPS necessitou a menos para que o usuário estivesse em melhores condições para voltar ao seu cotidiano. Considera-se que o processo de cuidado longitudinal que os usuários têm no CAPS, além de ter seu PTS construído de forma participativa e conjunta por usuário, sua rede social/família e profissionais contribui para que o tempo de acolhimento seja menor do que uma internação hospitalar. Os CAPS têm tido importante papel para diminuir o número de internações em hospitais psiquiátricos, uma vez que podem acompanhar intensivamente os usuários, evitando situações de crise, assim como podem também acompanhar estas situações sem precisar interná-los (Santos et al., 2013). Embora o CAPS venha dando conta de diminuir a frequência de crise dos usuários, salienta-se que crises são inevitáveis e quando acontecerem é de extrema importância que existam CAPS que possam dar conta deste momento na vida dos usuários sem que seja necessário interná-los. Sendo assim, é fundamental o investimento em CAPS III.

Segundo Nicacio e Campos (2004), o acolhimento à crise é um trabalho artesanal e que depende de conversa entre todos os envolvidos no processo de cuidado: usuário, rede de apoio e profissionais da saúde. Deve-se saber que o acolhimento sempre trará momentos de tensão, mas faz parte do trabalho no CAPS aprender a lidar com esta situação e negociar com o usuário que está em sofrimento. Ainda conforme os autores, considerando que o acolhimento não é tutelar restringindo, mas sim garantindo direitos, causa estranhamento observar que uma das categorias dos motivos de saída do acolhimento é a "evasão", principalmente pensando que o CAPS é um serviço porta aberta. O trabalho, principalmente em momentos de crise, deve ser construído na base da relação, do vínculo, da negociação e do estar junto, contornando-se assim possíveis saídas sem retorno e pensando no melhor 
cuidado ao usuário neste momento mais vulnerável que é a crise. É importante observar que a necessidade de transferência para outro serviço de saúde foi pequena, indicando que o CAPS foi capaz de lidar com quase a totalidade das crises acolhidas dentro do próprio serviço. O estudo de Silva, Santos, Campelo, Cardoso, Silva e Peres (2020) traz o relato de uma equipe de enfermagem de um CAPS III acerca da importância e diferença do acolhimento noturno com a internação hospital, sendo o acolhimento um espaço para estar mais próximo do usuário que necessita de cuidado no momento de crise e que pode ser ouvido e acompanhado pela equipe que já o conhece e dará seguimento ao cuidado. Pensar que a maioria dos acolhimentos terminou devido à alta dos usuários remete à viabilidade de "viver sem manicômios" e à construção de novas possibilidades para as situações de crise (Nicacio \& Campos, 2004).

A Portaria n 336/02 ao dispor sobre o uso do acolhimento determina a quantidade de dias máxima para esta estratégia. No entanto, deve-se lembrar que o acolhimento noturno deve ser um recurso a ser utilizado de forma singular e contextualizada, assim como ser produzido como direito (Nicacio \& Campos, 2004). O trabalho no CAPS é feito nos preceitos da Reabilitação Psicossocial, propostos por Saraceno (2001), e pensado de forma a proporcionar independência e autonomia aos usuários e que estes sofram o menos possível; sendo assim, tentar-se-á de toda forma que o usuário esteja em crise por menos vezes e pelo tempo mínimo necessário. Para isso, é importante que o acolhimento noturno possa ser utilizado como estratégia no cuidado dos usuários e que os profissionais do CAPS tenham autonomia para, junto do usuário e de sua rede de apoio, decidirem se este recurso deve ou não ser utilizado.

Por fim, este estudo apresentou limitações ao não ser possível fazer alguns cruzamentos de variáveis, pois houve um número considerável de dados ausentes ou mesmo ilegíveis e incompletos nos prontuários e nos registros do documento analisado. Esta limitação foi apresentada também nos estudos de Santos et al. (2013), Belletini \& Gomes (2013) e Pereira et al. (2012), o que reforça a necessidade do registro adequado pelos profissionais nos prontuários dos usuários dos serviços de saúde. Outra limitação deu-se pelo fato de esta pesquisa ter sido realizada em apenas um CAPS do município.

\section{Considerações Finais}

Este estudo possibilitou traçar o perfil sociodemográfico dos usuários que utilizaram o acolhimento noturno e características de como esta estratégia de cuidado vem sendo utilizada num CAPS III. Identificou-se que a maioria dos usuários, com os dados informados, que utilizou o acolhimento noturno foi do sexo feminino, de cor branca, com escolaridade até o ensino médio, beneficiário do INSS, dos 31 aos 59 anos, com diagnóstico de esquizofrenia, transtornos esquizotípicos e transtornos delirantes. O acolhimento noturno foi utilizado, em sua maioria, no máximo cinco vezes com duração de sete dias. Mulheres ficaram mais vezes em acolhimento, no entanto, ficaram menos dias que os homens. Os usuários saíram do acolhimento, na maior parte, devido à alta.

Percebeu-se como limitação maior deste estudo a falta de informações sobre os usuários no documento analisado e, principalmente, nos prontuários. Destaca-se a relevância de orientação aos profissionais sobre a importância de registro em prontuário para pesquisas futuras e também para circulação de informação sobre o usuário para que este possa ser atendido sempre que necessário, não dependendo apenas dos profissionais de referência.

Propõe-se que o documento referente ao acolhimento noturno seja complementado com dados como "motivo de entrada" e "motivo de saída" para que os profissionais possam descrever as situações de forma singularizada e qualitativa. Ainda propomos que o preenchimento do documento seja realizado pela equipe como um todo, que pode ter mais informações sobre o usuário por conhecê-lo melhor e descentraliza o trabalho direcionado apenas à equipe de enfermagem, como é feito atualmente.

$\mathrm{O}$ estudo pretendeu contribuir para o compartilhamento de informação sobre uma estratégia de cuidado ainda pouco estudada, uma vez que há baixo número de CAPS III no Brasil. Entende-se que é fundamental que o acolhimento noturno seja visto como a importante forma de cuidado que é e que seja investido pelo poder público a fim de que o cuidado ofertado aos 
usuários de saúde mental possa ser feito em liberdade e no território, caminhando, desta forma, cada vez mais em direção à cidadania e assegurando-lhes direitos.

Sugere-se que sejam realizados mais estudos para compartilhar experiências já existentes sobre o acolhimento noturno de usuários e que profissionais de CAPS sejam escutados sobre quais as fragilidades, potências e melhorias a serem feitas para que este dispositivo de cuidado seja aprimorado e cada vez mais difundido a fim de evitar internações em hospitais psiquiátricos.

\section{Referências}

Amarante, P. (Coord.). (2013). Loucos pela Vida: a Trajetória da Reforma Psiquiátrica no Brasil (6a ed.). SDE/ENSP, FIOCRUZ

Balarin, M. L. G. S., Miranda, I. M. S. \& Carvalho, C. M. (2011). Centro de Atenção Psicossocial do município de Campinas: estudo sobre o perfil sociodemográfico e clínico de seus usuários. Revista de Ciências Médicas, 20, (3-4, 59-67).

Belletini, F. E. \& Gomes, K. M. (2013) Perfil dos usuários do Centro de Atenção Psicossocial e do Programa de Saúde Mental no município de Orleans - SC. Cadernos Brasileiros de Saúde Mental, Florianópolis, 5, (12, 161-175).

Bezerra Jr., B. C. (2007). Desafios da reforma psiquiátrica no Brasil. Physis, 17, 243-250.

Brasil. Direção-Geral da Sáude/OMS. (2002). Relatório Sobre a Saúde no Mundo (2001) - Saúde mental: nova concepção, nova esperança. Brasília: Climepsi Editores.

Costa, R. C., Coelho, M. O., Neto, E. M. R., Marques, L. A. R. V. \& Lotif, M. A. L. (2015). Perfil epidemiológico de usuários intensivos de um Centro de Atenção Psicossocial. Rev de Enfer UFPE Online, Recife, 9 (2), edição suplementar, p. 820-829.

Dell'Acqua, G \& Mezzina, R. (1991). Resposta à crise. In: Delgado, J. A loucura na sala de jantar. 53-79

Farnettane, A. T. (2016). Recolhimento não, acolhimento sim - Centro de Atenção Psicossocial - CAPS III - João Ferreira da Silva Filho - Complexo do Alemão - Rio de Janeiro/Brasil. Academus Revista Científica da Saúde. Secretaria Municipal de Saúde do Rio de Janeiro.

Gallio, G. \& Mezzina, R. (1988). Il funzionamento del servizio psichiatrico territoriale sulle 24 ore. In: Righetti, A. (A cura di). La questione psichiatrica. Pordenone: Centro Studi per la Ricerca, nel Campo della Salute Mentale. 209-221.

Gil, A. C. (2002). Como elaborar projetos de pesquisa/Antônio Carlos Gil. (4a ed.), Atlas.

Instituto Brasileiro de Geografia e Estatística - IBGE. (2016). Síntese de Indicadores Sociais - SIS.

Instituto Brasileiro de Geografia e Estatística - IBGE. (2018) Pesquisa Nacional por Amostra de Domicílios Contínua - PNAD Contínua.

Kinoshita, R. T. (2009). Saúde mental e antipsiquiatria em Santos: vinte anos depois. Caderno Brasileiro de Saúde Mental,1 (1).

Kinoshita, R. T. (2016). Contratualidade e reabilitação psicossocial. In:Pitta, A. (Org.) Reabilitação Psicossocial no Brasil. (4a ed.), Hucitec.

Machado, V. C. \& Santos, M. A. (2011). Taxa de permanência hospitalar de pacientes reinternados em hospital psiquiátrico. J Bras Psiquiatr, 60(1), 16-22.

Marconi, M. A.\& Lakatos, E. M. (2011). Metodologia do trabalho científico: procedimentos básicos, pesquisa bibliográfica, projeto e relatório, publicações e trabalhoscientíficos. (7a ed.), Atlas.

Ministério da Saúde. Lei $n^{o}$ 10.216, de 6 de abril de 2001. Dispõe sobre a proteção e os direitos das pessoas portadoras de transtornos mentais e refireciona o modelo assistencial em saúde mental. http://www.planalto.gov.br/ccivil_03/LEIS/LEIS_2001/L10216.htm

Ministério da Saúde. Portaria GM/MS n 336, de 19 de fevereiro de 2002. Estabelece que os Centros de Atenção Psicossocial poderão constituir-se nas seguintes modalidades de serviços: CAPS I, CAPS II e CAPS III, definidos por ordem crescente de porte/complexidade e abrangência populacional. http://bvsms.saude.gov.br/bvs/saudelegis/gm/2002/prt0336_19_02_2002.html

Minitério da Saúde. Portaria $n^{\circ} 854$, de 22 de agosto de 2012. Inclui, altera e exclui procedimentos da na Tabela de Procedimentos, Medicamentos, Órteses, Próteses e Materiais Especiais do Sistema Único de Saúde. http://bvsms.saude.gov.br/bvs/saudelegis/sas/2012/prt0854_22_08_2012.html

Ministério da Saúde. (2007). Política Nacional de Saúde Integral da População Negra.

Nicacio, F. \& Campos, G. W. S. (2004). A complexidade da atenção às situações de crise: contribuições da desinstitucionalização para a invenção de práticas inovadoras em saúde mental. Revista de Terapia Ocupacional da Universidade de São Paulo, 15, 71-81.

Pelisoli, C. L. \& Moreira, A. K. (2005). Caracterização epidemiológica dos usuários do Centro de Atenção Psicossocial Casa Aberta. Revista Psiquiátrica do Rio Grande do Sul, 27 (3), 270-277.

Pelisoli, C. L. \& Moreira, A. K. (2007). Avaliação de um centro de atenção psicossocial por meio do perfil de seus usuários. Mental, Barbacena, 5 (8), 61-75.

Pereira, M. O., Souza, J. M., Costa, A. M., Vargas, D., Oliveira, M. A. F. \& Moura, W. N. (2012). Perfil dos usuários de Serviços de Saúde Mental do município de Lorena - São Paulo. Acta Paulista de Enfermagem, 25 (1), 48-54. 
Research, Society and Development, v. 10, n. 10, e164101018758, 2021

(CC BY 4.0) | ISSN 2525-3409 | DOI: http://dx.doi.org/10.33448/rsd-v10i10.18758

Prodanov, C. C. (2013). Metodologia do trabalho científico [recurso eletrônico]: métodos e técnicas da pesquisa e do trabalho acadêmico. (2a ed.). Novo Hamburgo: Feevale.

Santos, E. G. \& Siqueira, M. M. (2010). Prevalência dos Transtornos Mentais na população adulta Brasileira: uma revisão sistemática de 1997 a 2009. J Bras Psiquiatr, 59, 238/3-247.

Santos, G. F., Nascimento, Y. C. M. L., Veríssimo, R. C. S. S., Cavalcante, J. C., Brêda, M. Z. \& Holanda, J. B. L. (2013). O perfil epidemiológico dos usuários de um Centro de Atenção Psicossocial. Rev de Enfer UFPE Online, Recife, 7 (3), 679-687.

Saraceno, B. (1994). Manual de saúde mental. Hucitec.

Saraceno, B. (2001). Libertando identidades: da reabilitação psicossocial à cidadania possível. Te Corá/Instituto Franco Basaglia.

Silva, A. L. A. (2012). A construção de um projeto de extensão universitária no contexto das políticas públicas: saúde mental e economia solidária. Tese (Livre Docência). Escola de Enfermagem da Universidade de São Paulo.

Silva, N. G., Barros, S., Azevedo, F. C., Batista, L. E. \& Policarpo, V.C. (2017). O quesito raça/cor nos estudos de caracterização de usuários de Centro de Atenção Psicossocial. Saúde e Sociedade, 26, 100-114.

Silva, S. N., Lima, M. G. \& Ruas, C. M. (2020). Uso de medicamentos nos Centros de Atenção Psicossocial: análise das prescrições e perfil dos usuários em diferentes modalidades do serviço. Ciência \& Saúde Coletiva, 25(7):2871-2882.

Silva, T. C. S., Santos, T. M., Campelo, I. G. M. T., Cardoso, M. M. V. N., Silva, A. D. \& Peres, M. A. A. (2020). Acolhimento noturno em um Centro de Atenção Psicossocial III. Rev Bras Enferm;73(1).

Souza, R. F. \& Padula, M. P. C. (2020). Condições de pessoas em sofrimento psíquico acompanhadas em Centro de Atenção Psicossocial (CAPS) e internadas em hospitais. Braz. J. Hea. Rev., 3(5), 11967-11988.

Zanello, V., Fiuza, G. \& Costa, H. S. (2015). Saúde mental e gênero: facetas gendradas do sofrimento psíquico. Fractal, 27, 238-246. 